\title{
Too Much of a Good Thing: Endogenous Business Cycles generated by Bounded Technological Progress
}

\begin{abstract}
Orlando Gomes*
Escola Superior de Comunicação Social [Instituto Politécnico de Lisboa] and Unidade de Investigação em Desenvolvimento Empresarial [UNIDE/ISCTE].
\end{abstract}

- July, 2006 -

\begin{abstract}
Following Jones and Williams (2000), we assume that R\&D is simultaneously subject to positive and to negative external effects (e.g., the non rival nature of technology conflicts with congestion externalities). This observation allows to conceive an economy where two R\&D sectors evolve without departing significantly from each other in terms of their productive results (society tends to penalize imbalances in technical progress, making negative external effects to appear associated to a sector when this outstands relatively to the other sector; the second sector, in turn, will be subject to positive externalities that reflect a catching up effect). The proposed framework, when associated to a growth setup, is able to replicate the existence of endogenous fluctuations and, therefore, it intends to be a contribution to the literature on endogenous business cycles.
\end{abstract}

Keywords: Technology, Externalities, Endogenous business cycles, Growth models, Nonlinear dynamics and chaos.

JEL classification: C61, E32, O41

\footnotetext{
* Orlando Gomes; address: Escola Superior de Comunicação Social, Campus de Benfica do IPL, 1549014 Lisbon, Portugal. Phone number: + 3519334209 15; fax: + 351217162 540. E-mail: ogomes@escs.ipl.pt.

Acknowledgements: Financial support from the Fundação Ciência e Tecnologia, Lisbon, is grateful acknowledged, under the contract No POCTI/ECO/48628/2002, partially funded by the European Regional Development Fund (ERDF). I would like to thank, as well, Vivaldo Mendes for helpful discussion and suggestions.
} 


\section{Introduction}

An insightful analysis made by Jones and Williams (2000) have called the attention for an important fact about investment in $R \& D$ and its implications to the perception we have about economic growth. As it is well known, frequently we observe positive externalities associated to the non-rival nature of technology and knowledge, and this observation makes us jump to the conclusion that there is an economy wide receptiveness to innovations: households will be eager to gain access to goods that are technologically more sophisticated, the government protects and stimulates private investors in $\mathrm{R} \& \mathrm{D}$ and other economic sectors tend to provide the environment needed to $R \& D$ to be developed to its fullest capacity. The two cited authors emphasize the relevance of these positive externalities, but they highlight as well that negative externalities associated to the R\&D sector may also be identified. Namely, the congestion external effect provoked by patent racing and the eventually too fast obsolescence of previous generations of knowledge goods act as forces that prevent society from supporting an accelerated development of new technologies. If economic agents perceive technical progress as faster than what they can keep up to, then households will lose interest in innovation, the government will attribute less subsidies to research and business partners will prefer to continue to explore the existent wave of technology rather than stimulating a new one.

The previous argument can be reinforced with the well known 'productivity paradox' of Solow (1987) [see Brynjolfsson (1993), Bresnahan, Brynjolfsson and Hitt (2002) and Gunnarsson, Mellander and Savvidou (2004), among many others]. The paradox essentially states that innovation in the computer and software industries did not gave place to important productivity gains. Some of the explanations for the paradox (that were put forward in the papers cited above) focus on the idea that the information and communication technology (ICT) revolution was not followed by other complementary innovations, for example related with human capital formation and with the design and structure of organizations; as a result, the ICT were rejected by many organizations, who could not find much use in them [for instance, Bresnahan, Brynjolfsson and Hitt (2002) found that firms adopting ICT without any other innovation investment became less productive than the firms maintaining traditional methods of production]. This is a good example of how a dynamic R\&D sector can suffer a negative externality if it is not accompanied by other technical developments. The truth is that the receptiveness to innovations is linked to a balanced evolution of the 
various $R \& D$ sectors, in such a way that one can conceive an economy wide negative external effect affecting the leader R\&D producers and, simultaneously, a positive external effect that allows the follower R\&D firms to catch up with the first ones (in the case of the ICT, we can think about computers as stimulating human capital upgrading and organizational remodelling that without such communication technologies revolution would never have happened; this is the positive external effect).

Having in mind that positive and negative externalities co-exist in what respects technological progress, we propose a model of growth with two R\&D sectors. The main feature of this discrete time model is that the outcome of the two sectors should not depart too much from each other, in the sense that the society does not tolerate technological imbalances. Thus, if the output of $\mathrm{R} \& \mathrm{D}$ sector $A$ is higher than the output of $\mathrm{R} \& \mathrm{D}$ sector $B$, a negative externality to the production of knowledge arises in sector $A$ and, simultaneously, a positive externality can be found in sector $B$, and the other way around if in a given moment the second sector is the more productive one. With this framework, we will be able to justify the presence of endogenous business cycles in a conventional economic growth framework.

This work intends to contribute to the literature that attempts to explain cycles under a deterministic growth model, and thus it approaches the analysis undertaken, among others, by Christiano and Harrison (1999), Schmitt-Grohé (2000), Guo and Lansing (2002), Cellarier (2006) and Gomes (2006a). The novelty, relatively to the referred papers, is that cycles are not determined by final goods sector externalities, specific conditions of the labour market, learning mechanisms or conditions of demand, but, as stated, by the simultaneous presence of positive and negative externalities in the production of knowledge, which produce a tendency for different $R \& D$ sectors to evolve at a similar pace.

Furthermore, one should stress that the analysis is undertaken under the conventional growth setup, i.e., resorting to a competitive economy environment, where the several production functions to assume exhibit constant returns to scale. In this way, the search for non linear dynamics is made under a framework that approaches the one that is taken, among others, by Nishimura and Yano (1995) and Boldrin, Nishimura, Shigoka and Yano (2001), that is, the standard Walrasian growth model. This approach neglects market inefficiencies of the Keynesian type (nominal rigidities, price stickiness, strategic complementarities and coordination failures) and departs also from the Real Business Cycles (RBC) theory, since the analysis is fully deterministic: there are no stochastic variables underneath the fluctuations; these are generated by purely 
deterministic relations. Nonlinear deterministic dynamics were first addressed in economics, and more precisely with regard to business cycles, with an influent strand of theory introduced along the 1980s and early 1990s: Stutzer (1980), Benhabib and Day (1981), Day (1982), Grandmont (1985), Baumol and Benhabib (1989), Boldrin and Woodford (1990), Chiarella (1992) and Bullard and Butler (1993), just to cite some of the most prominent contributions.

The paper is organized as follows. Section 2 presents the model; section 3 characterizes the dynamics underlying the two-sector R\&D activity, when externalities are present. First, local dynamics in the steady state vicinity are addressed, and, on a second moment, we discuss the global properties of the dynamic system; we will be particularly concerned with the chaotic features of the model; section 4 introduces a capital accumulation constraint to further characterize the economic implications of the proposed setup; finally, section 5 concludes.

\section{The Model}

We consider an economy populated by infinitely lived agents where human capital, $H_{t}$, grows at a constant rate $\gamma>0$, that is, $H_{t+1}-H_{t}=\gamma H_{t}, H_{0}$ given. In this economy there are two technological sectors $(A$ and $B)$. In the absence of external effects, these sectors can be described by accumulation processes similar to the ones generally presented in growth models [see, e.g., Romer (1990) and Jones (1995)]: $A_{t+1}-A_{t}=f^{1}\left(A_{t}, H_{t}\right)-\rho A_{t}, A_{0}$ given, and $B_{t+1}-B_{t}=f^{2}\left(B_{t}, H_{t}\right)-\sigma B_{t}, B_{0}$ given, with $\rho>0$ and $\sigma>0$ obsolescence rates. Cobb-Douglas production functions are assumed: $f^{1}\left(A_{t}, H_{t}\right)=g_{1} A_{t}^{\phi}\left(u H_{t}\right)^{1-\phi}$ and $f^{2}\left(B_{t}, H_{t}\right)=g_{2} B_{t}^{\mu}\left[(1-u) \cdot H_{t}\right]^{1-\mu}$. Parameter $u$ is the share of human capital used to produce technology good $A ; g_{1}$ and $g_{2}$ are positive values and $\phi, \mu \in(0,1)$.

Under diminishing marginal returns to technology and human capital, the dynamics of the above system are easily identifiable. In concrete, a unique stable steady state is attainable, independently of the initial state. This means that, like human capital, both $\mathrm{R} \& \mathrm{D}$ variables will grow in the long run at the same rate $\gamma$. Constant technology human capital ratios would define the steady state locus: $\frac{\bar{A}}{\bar{H}}=u \cdot\left(\frac{g_{1}}{\rho}\right)^{1 /(1-\phi)}$ and 
$\frac{\bar{B}}{\bar{H}}=(1-u) \cdot\left(\frac{g_{2}}{\sigma}\right)^{1 /(1-\mu)}$. These results are trivial in the literature about endogenous growth and they are mainly the outcome imposed by the perfectly competitive market structure that this setup implicitly considers.

Now, take the arguments in the introduction, i.e., assume that if, in a given moment, sector $A$ generates a larger output than $\operatorname{sector} B$, then a positive externality will be associated with sector $B$ and a negative externality will be present in sector $A$ (and the other way around, if sector $B$ is the dominant sector in a given time period). We modify the R\&D equations to include the externalities: $A_{t+1}-A_{t}=f^{1}\left(A_{t}, H_{t}\right) \cdot \xi^{1}\left(A_{t}, B_{t}\right)-\rho A_{t}$ and $B_{t+1}-B_{t}=f^{2}\left(B_{t}, H_{t}\right) \cdot \xi^{2}\left(A_{t}, B_{t}\right)-\sigma B_{t}$.

The externality terms are defined as follows: $\xi^{1}\left(A_{t}, B_{t}\right)=1+\theta_{1} \cdot \arctan \left(\frac{B_{t}-A_{t}}{H_{t}}\right)$ and $\xi^{2}\left(A_{t}, B_{t}\right)=1+\theta_{2} \cdot \arctan \left(\frac{A_{t}-B_{t}}{H_{t}}\right)$. Parameters $\theta_{1}$ and $\theta_{2}$ are positive constants. The shape of the externality functions serves our purposes: if $A_{t}=B_{t}$ then no externalities exist and we have a conventional competitive model. When the two technology variables have different values, function $\xi, i=1,2$, will represent a quantity below or above one, depending on the level of technology of the sector being above or below the other sector's technology level, which reflects, respectively, the presence of negative or positive externalities related to each $R \& D$ sector.

The externality function for sector $A$ is represented in figure 1 . We observe that the externality is positive (the term associated to the production function is larger than 1) when the output of the considered technological level is lower than the output of the other $R \& D$ sector, and that the externality is negative (the term associated to the production function is smaller than 1) when the output of the considered technological level is above the output of the other R\&D sector.

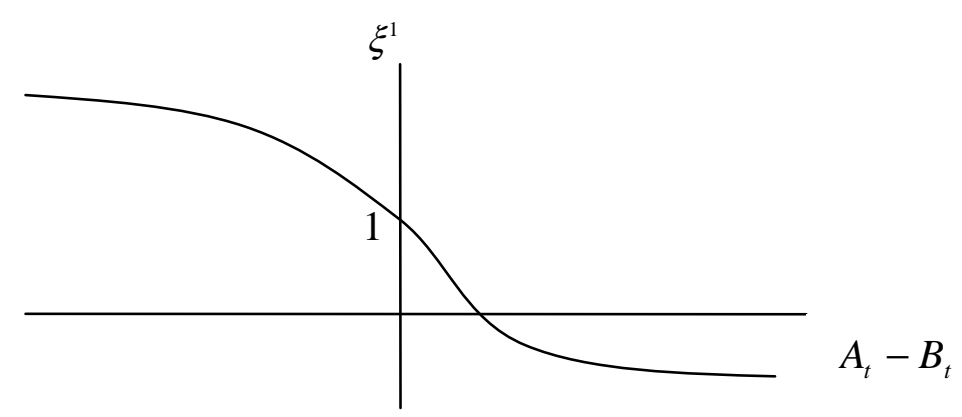


Figure 1 - Externality function for R\&D sector $A$.

Defining $a_{t} \equiv \frac{A_{t}}{H_{t}}$ and $b_{t} \equiv \frac{B_{t}}{H_{t}}$, the problem can be presented in intensive form, $\left\{\begin{array}{l}a_{t+1}=\psi_{1} a_{t}^{\phi} \cdot \xi^{1}\left(a_{t}, b_{t}\right)+\psi_{2} a_{t} \\ b_{t+1}=\varphi_{1} b_{t}^{\mu} \cdot \xi^{2}\left(a_{t}, b_{t}\right)+\varphi_{2} b_{t}\end{array} \quad \quad\right.$ with $\quad \xi^{1}\left(a_{t}, b_{t}\right)=1+\theta_{1} \cdot \arctan \left(b_{t}-a_{t}\right)$, $\xi^{2}\left(a_{t}, b_{t}\right)=1+\theta_{2} \cdot \arctan \left(a_{t}-b_{t}\right), \quad \psi_{1}=\frac{g_{1} u^{1-\phi}}{1+\gamma}, \quad \psi_{2}=\frac{1-\rho}{1+\gamma}, \quad \varphi_{1}=\frac{g_{2} \cdot(1-u)^{1-\mu}}{1+\gamma}$ and $\varphi_{2}=\frac{1-\sigma}{1+\gamma}$

The previous system produces nonlinear dynamic results, that are explored in the next section.

\section{Dynamics}

Local stability properties of the two-sector R\&D problem with externalities are first addressed. Subsequently, we take a look at possible global dynamics outcomes.

\subsection{Local Analysis}

To study local dynamics one should determine the steady state pair $(\bar{a}, \bar{b})$. This pair is the solution of the system,

$$
\left\{\begin{array}{l}
\arctan (\bar{b}-\bar{a})=\frac{1-\psi_{2}}{\theta_{1} \cdot \psi_{1}} \cdot \bar{a}^{1-\phi}-\frac{1}{\theta_{1}} \\
\arctan (\bar{a}-\bar{b})=\frac{1-\varphi_{2}}{\theta_{2} \cdot \varphi_{1}} \cdot \bar{b}^{1-\mu}-\frac{1}{\theta_{2}}
\end{array} ; \text { since the function } \arctan (.)\right. \text { is symmetric }
$$

around zero, the condition $\arctan (\bar{b}-\bar{a})+\arctan (\bar{a}-\bar{b})=0$ is true and can be used to obtain long run constant values for the variables. Although the computation does not allow to get explicit values $(\bar{a}, \bar{b})$ unless specific parameter values are attributed and an extrapolation process is used, it is straightforward to perceive that the equilibrium exists and it is unique. In what follows, we will use the pair of positive values $(\bar{a}, \bar{b})$ as it is, without presenting it as combinations of parameters.

The linearization of the $R \& D$ equations in the steady state vicinity yields the following matrix system, 


$$
\left[\begin{array}{c}
a_{t+1}-\bar{a} \\
b_{t+1}-\bar{b}
\end{array}\right]=\left[\begin{array}{cc}
\eta_{1}-\omega_{1} & \omega_{1} \\
\omega_{2} & \eta_{2}-\omega_{2}
\end{array}\right] \cdot\left[\begin{array}{c}
a_{t}-\bar{a} \\
b_{t}-\bar{b}
\end{array}\right]
$$

with $\omega_{1} \equiv \frac{\psi_{1} \cdot \theta_{1} \cdot \bar{a}^{\phi}}{1+(\bar{b}-\bar{a})^{2}}, \quad \omega_{2} \equiv \frac{\varphi_{1} \cdot \theta_{2} \cdot \bar{b}^{\mu}}{1+(\bar{a}-\bar{b})^{2}}, \eta_{1} \equiv \phi \cdot\left(1-\psi_{2}\right)+\psi_{2}, \eta_{2} \equiv \mu \cdot\left(1-\varphi_{2}\right)+\varphi_{2}$ all positive quantities.

Conditions for stability, that is, conditions under which the eigenvalues of the Jacobian matrix are inside the unit circle, can be given in terms of the trace and determinant of this matrix (that we designate by $J$ ), as follows

$$
\begin{aligned}
& 1+\operatorname{Tr}(J)+\operatorname{Det}(J)>0 \Rightarrow 1+\eta_{1}+\eta_{2}+\eta_{1} \eta_{2}>\omega_{1}+\omega_{2}+\omega_{1} \eta_{2}+\omega_{2} \eta_{1} \\
& 1-\operatorname{Tr}(J)+\operatorname{Det}(J)>0 \Rightarrow 1+\omega_{1}+\omega_{2}+\eta_{1} \eta_{2}>\eta_{1}+\eta_{2}+\omega_{1} \eta_{2}+\omega_{2} \eta_{1} \\
& 1-\operatorname{Det}(J)>0 \Rightarrow 1+\omega_{1} \eta_{2}+\omega_{2} \eta_{1}>\eta_{1} \eta_{2}
\end{aligned}
$$

To guarantee diminishing marginal returns in each one of the production functions, the following constraints apply, $\eta_{1}<1$ and $\eta_{2}<1$. Under these two conditions, the second and third presented inequalities are always satisfied. Only the first one can be a true or a false relation for different sets of parameter values. Note, as well, that complex roots for the characteristic equation are excluded, and thus both eigenvalues are real numbers; this is true given that: $\operatorname{Tr}(J)^{2}-4 \operatorname{Det}(J)=\left(\omega_{1}-\omega_{2}-\eta_{1}+\eta_{2}\right)^{2}+4 \omega_{1} \omega_{2}>0$.

Figure 2 draws the standard diagram of stability in $\mathbf{R}^{2}$. 


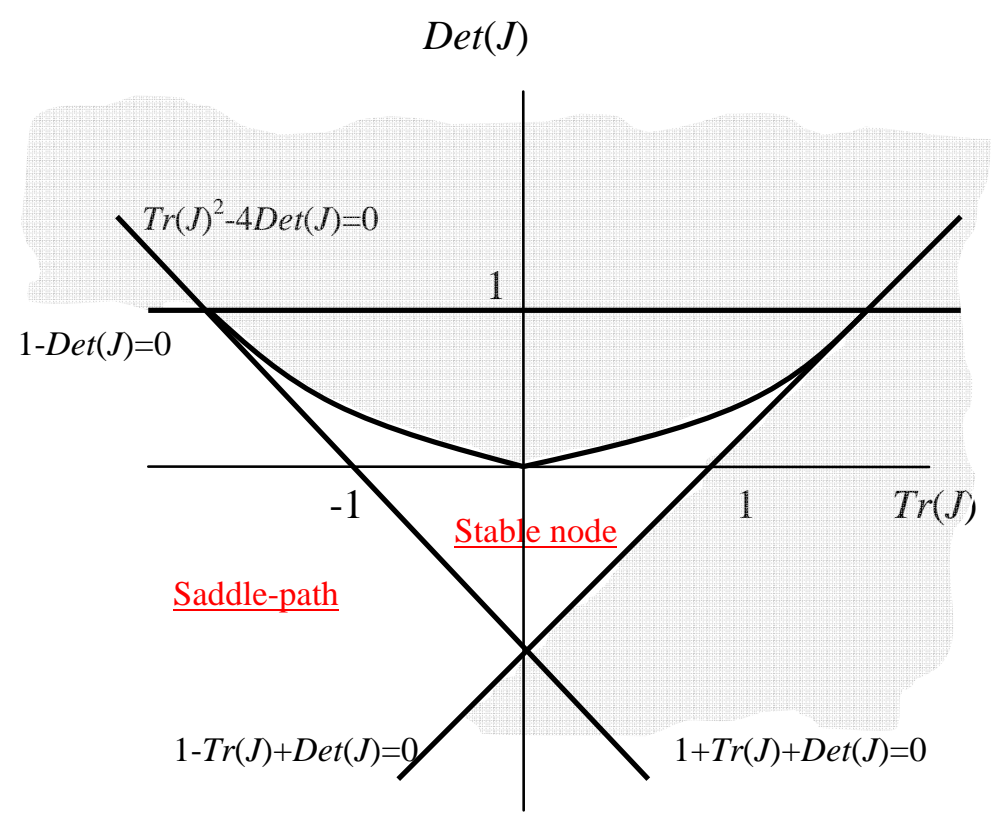

Figure 2 - Local stability dynamics.

As figure 2 allows to perceive there are only two types of results that are possible in what respects local dynamics. The stable node area corresponds to the case where condition $1+\operatorname{Tr}(J)+\operatorname{Det}(J)>0$ is satisfied. The area of saddle-path stability is accomplished for $1+\operatorname{Tr}(J)+\operatorname{Det}(J)<0$. The areas in grey are not possible stability locations for our system, because they correspond to regions where the other stability conditions are violated, what does not happen in our problem.

In what concerns bifurcation analysis, one concludes that Neimark-Sacker bifurcations are outside the scope of our system, because complex eigenvalues were excluded from the feasible set of outcomes; also, fold, pitchfork and transcritical bifurcations are impossible to obtain, given that condition $1-\operatorname{Tr}(J)+\operatorname{Det}(J)=0$ never holds. This leaves us with the possibility of a flip bifurcation, when the following condition is satisfied: $1+\eta_{1}+\eta_{2}+\eta_{1} \eta_{2}=\omega_{1}+\omega_{2}+\omega_{1} \eta_{2}+\omega_{2} \eta_{1}$. Combining the condition for a flip bifurcation with the others, always satisfied, stability conditions, one observes that the presence of a flip bifurcation requires that inequality $\omega_{1}+\omega_{2}>\eta_{1}+\eta_{2}$ must hold.

Our generic analysis of local stability can be synthesized in two main results:

(i) The R\&D system with externalities supports only two types of stability (stable node and saddle-path);

(ii) The transition between the two types of stability areas occurs through a flip bifurcation. 
Imposing some simplifying assumptions one can analyze further the local properties of the difference equations system. Consider the particular case in which $\eta_{1}=\eta_{2} \equiv \eta$. In this case, the eigenvalues can be presented through simple expressions and the analysis of stability can be made directly with them. The eigenvalues of the Jacobian matrix are, under the imposed constraint, $\lambda_{1}=\eta$ and $\lambda_{2}=\eta-\omega_{1}-\omega_{2}$. The first eigenvalue is always inside the unit circle; the second is inside the unit circle if the following condition is satisfied: $\omega_{1}+\omega_{2}>1+\eta$. This is the condition for stable node stability. If the inequality is of opposite sign, then saddle-path stability is observed. Figure 3 represents areas of stability in the space of parameters $\left(\omega_{1}, \omega_{2}\right)$. The line separating the two regions corresponds to the bifurcation; note that this line is as much far from the origin as the higher is the value of parameter $\eta$.

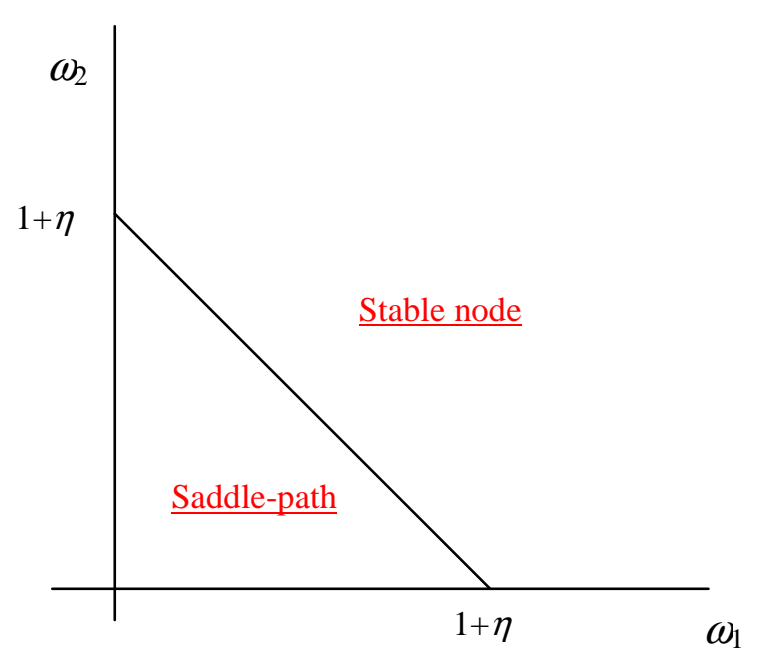

Figure 3 - Regions of stable node stability and saddle-path stability in the space of parameters.

To end our local dynamic analysis, we calculate the stable arm when saddle-path stability holds. To proceed with this computation, we determine an eigenvector associated to the eigenvalue inside the unit circle. We obtain $p=\left[\begin{array}{ll}1 & \frac{\eta_{1}-\omega_{1}-\lambda_{1}}{\omega_{1}}\end{array}\right.$. The second element of the eigenvector is the slope of the stable arm, that is, the stable trajectory can be written as $b_{t}-\bar{b}=\frac{\eta_{1}-\omega_{1}-\lambda_{1}}{\omega_{1}} \cdot\left(a_{t}-\bar{a}\right)$. The convergence to the equilibrium point is characterized by a same qualitative movement of both technology variables if the condition $\eta_{1}-\lambda_{1}>\omega_{1}$ is met. Otherwise, for $\eta_{1}-\lambda_{1}<\omega_{1}$, the adjustment to the steady state is described by an inverse relation between the evolution of technology variables: $b_{t}$ rises when $a_{t}$ declines, or the other way around. 
In the particular case $\eta_{1}=\eta_{2}$, the stable trajectory simplifies to $b_{t}-\bar{b}=\bar{a}-a_{t}$, that is, assuming that the stable trajectory is followed, a unit positive change in one of the $R \& D$ variables occurs simultaneously with a unit negative change on the other technology index.

Also the unstable trajectory can be computed; this is, on the general case, $b_{t}-\bar{b}=\frac{\eta_{1}-\omega_{1}-\lambda_{2}}{\omega_{1}} \cdot\left(a_{t}-\bar{a}\right)$ and, on the simplified version, $b_{t}-\bar{b}=\frac{\omega_{2}}{\omega_{1}} \cdot\left(a_{t}-\bar{a}\right)$. Figure 4 illustrates the dynamics of the saddle-path case (under the less general formulation of the problem); in this diagram, one identifies a positively sloped unstable arm and a negatively sloped stable trajectory that is followed only if the initial state of the system is placed over such stable trajectory.

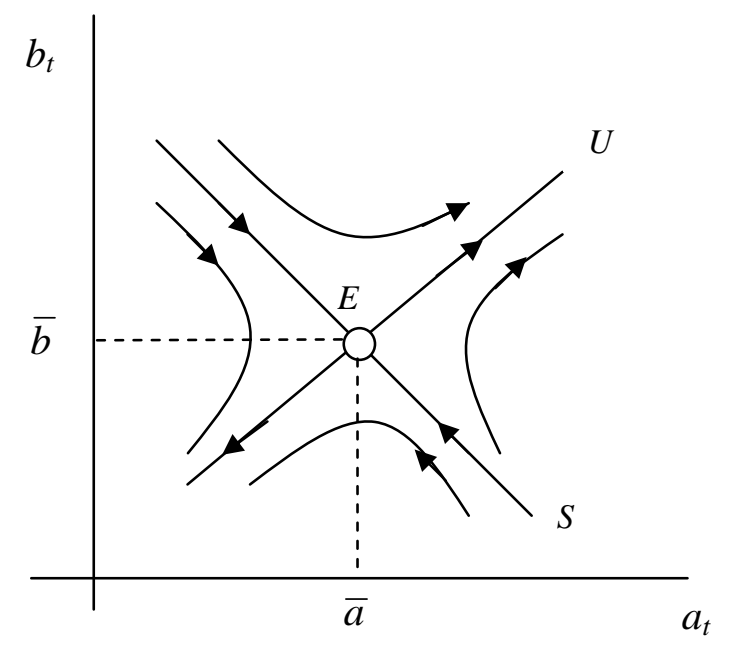

Figure 4 - Phase diagram in the saddle-path case.

Assuming that initial values of technology are below their steady state outcomes, instability means, according to figure 4, that technological conditions will progressively deteriorate. Under a policy point of view, the authorities have, in this case, the task of guaranteeing full stability; policy parameters should be manipulated in order to guarantee $\omega_{1}+\omega_{2}>1+\eta$, and in this way make it possible for the R\&D sectors to work in order to accomplish the correspondent steady state technology values.

\subsection{Global Dynamics}

The local analysis is misleading. While this can only make the separation between regions of stability and instability (more rigorously, saddle-path stability), a global 
dynamic analysis reveals a huge set of long term possibilities including periodic and aperiodic cycles. The analysis one can undertake at a global level is essentially graphical and based on numerical examples. For specific parameter values, we illustrate global dynamics with figures 5 to 11 . $^{1}$ The graphical presentations give just an illustration of the immense set of possible results, when parameter values are varied. In particular, it is important to emphasize that cycles of various orders are obtainable.

In figures 5 and 6 bifurcation diagrams are drawn; the selected bifurcation parameter is $\theta_{1}$, but choosing other bifurcation parameters (namely $\theta_{2}, \phi$, or $\mu$ ) would allow as well to obtain meaningful results. For the case in appreciation, we find for both variables that cycles of low order (order two or order four) alternate with cycles of higher order or without any identifiable order. Chaotic motion arises for some values of the chosen parameter, a result that will be reinforced with the following graphical presentations.

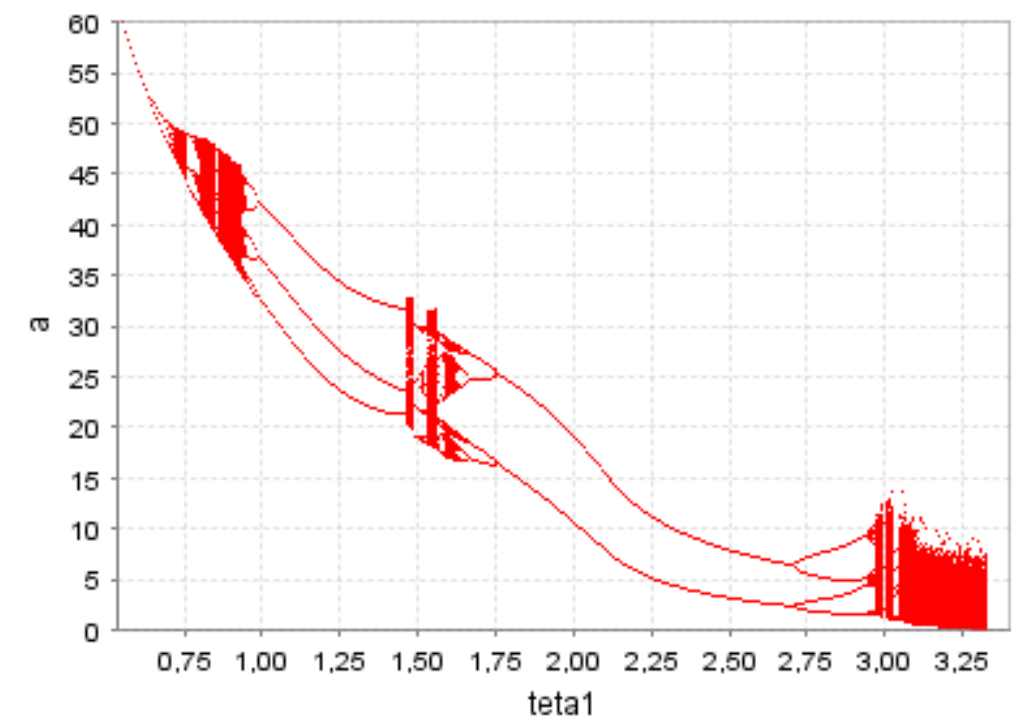

Figure 5 -Bifurcation diagram $\left(\theta_{1}, a_{t}\right)$

[parameter values $\psi_{1}=\varphi_{1}=0.5, \psi_{2}=0.95, \varphi_{2}=0.9, \phi=0.7, \mu=0.3, \theta_{2}=2$ ].

\footnotetext{
1 These figures, as well as figures 12 to 14, were drawn using iDMC (interactive Dynamical Model Calculator). This is a free software program available at www.dss.uniud.it/nonlinear, and copyright of Marji Lines and Alfredo Medio.
} 


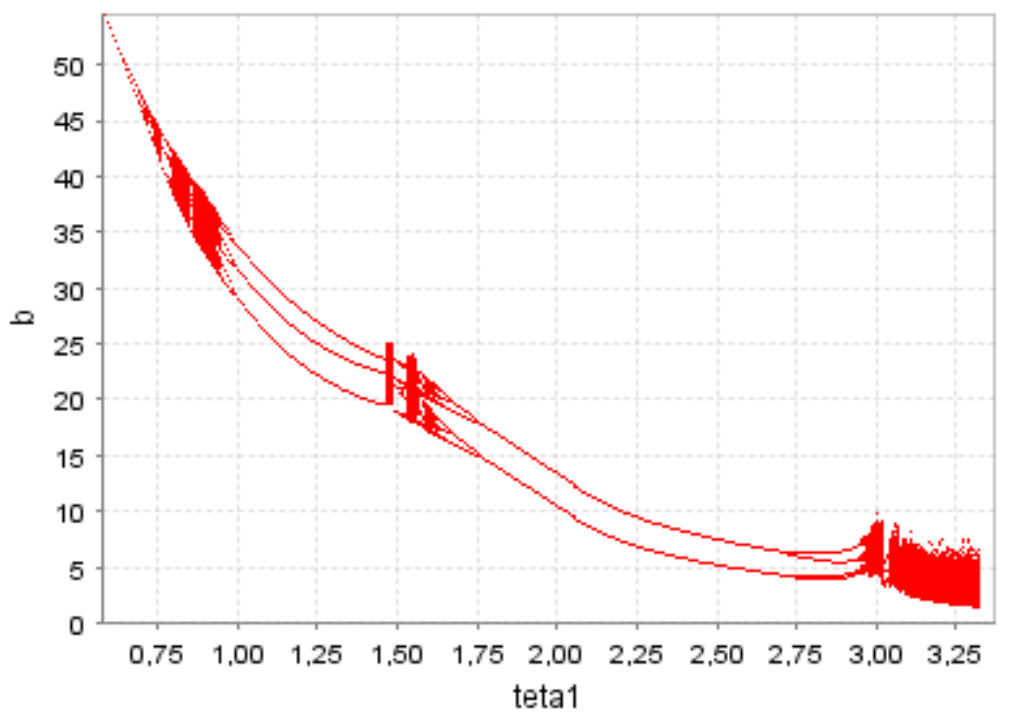

Figure 6 -Bifurcation diagram $\left(\theta_{1}, b_{t}\right)$

[parameter values $\psi_{1}=\varphi_{1}=0.5, \psi_{2}=0.95, \varphi_{2}=0.9, \phi=0.7, \mu=0.3, \theta_{2}=2$ ].

Figures 7 and 8 present diagrams that identify, with several colours, cycles of various orders in the space of parameters; in figure 7, one identifies different periodicities for the parameters associated with the externality terms; in figure 8, a same analysis is undertaken for the elasticities in the R\&D production functions. For both cases, one is able to confirm the richness of the dynamic results; for small changes in parameter values, the system passes from fixed point stability to cycles of low order, cycles of high periodicity and instability. The local analysis, where only fixed point and instability outcomes could be identified, did not allow for perceiving that in many cases strange dynamics effectively arise.

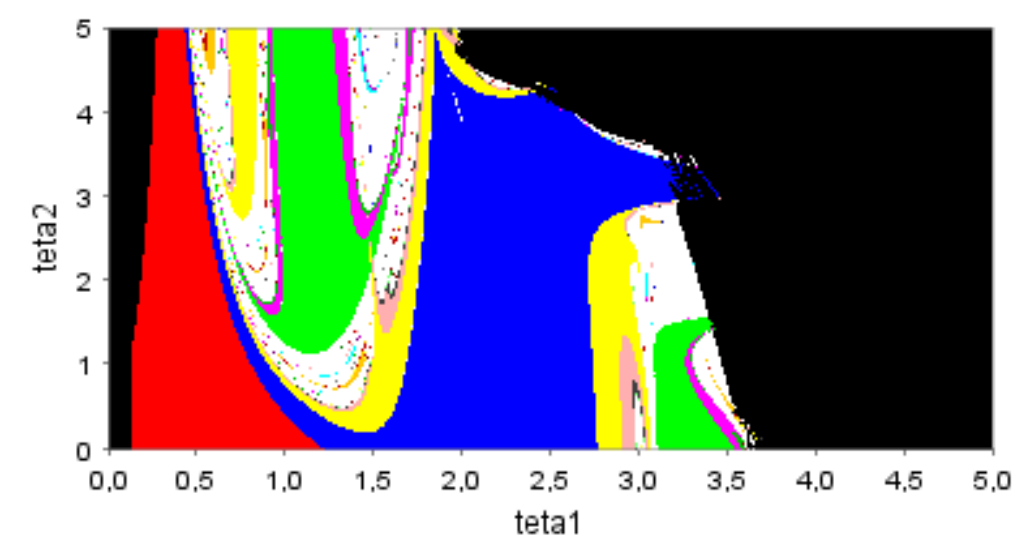

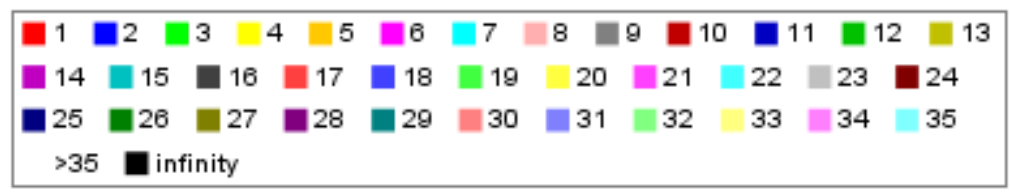

Figure 7 - Cycles in the space of parameters $\left(\theta_{1}, \theta_{2}\right)$ [parameter values $\left.\psi_{1}=\varphi_{1}=0.5, \psi_{2}=0.95, \varphi_{2}=0.9, \phi=0.7, \mu=0.3\right]$. 

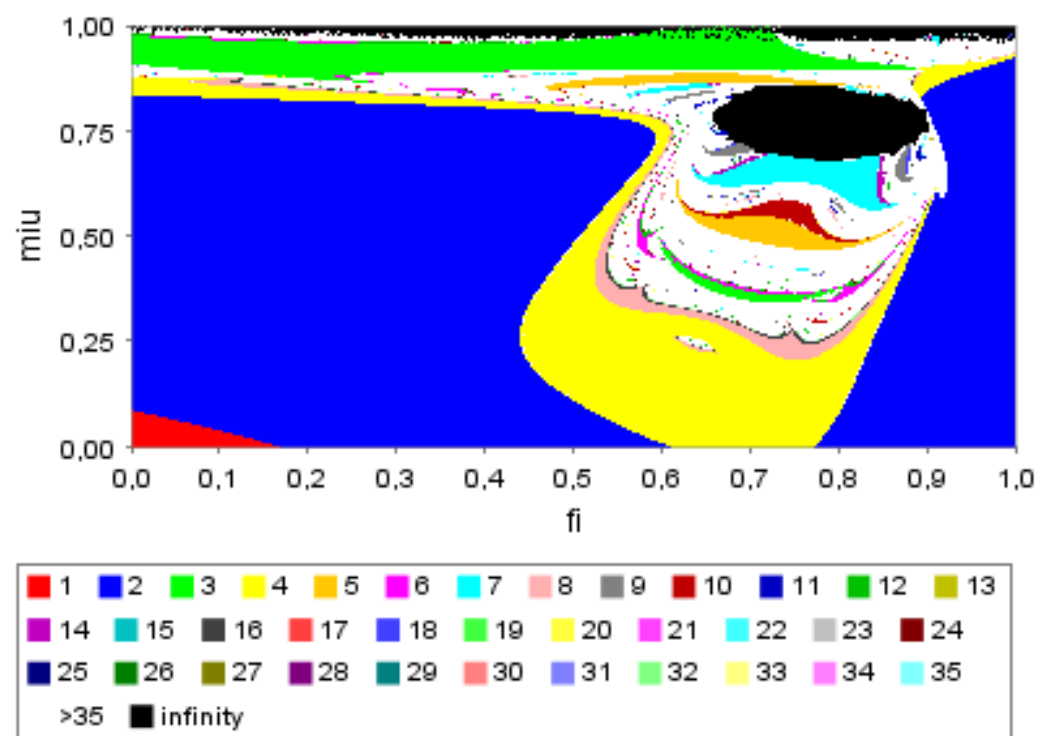

Figure 8 - Cycles in the space of parameters $(\phi, \mu)$ [parameter values $\left.\psi_{1}=\varphi_{1}=0.5, \psi_{2}=0.95, \varphi_{2}=0.9, \theta_{1}=1.53, \theta_{2}=2\right]$.

Finally, we consider a combination of parameter values that leads to cycles of no identifiable order (chaos) and present an attractor (figure 9) and the time series of both technology variables (figures 10 and 11). The chaotic motion is intuitively explained under the framework that was proposed; recall that sector $A$ suffers a negative externality when $a_{t}$ rises above $b_{t}$ and the opposite for sector $B$, and thus there is a permanent conflict between forces of accumulation and externalities that allow variables $a_{t}$ and $b_{t}$ not to be constant but to fluctuate around a constant value.

Recall, as well, that $a_{t}$ and $b_{t}$ do not grow systematically over time at a positive rate, but this does not mean that, on average, technical progress does not exist. Our intensive form technology variables are ratios between technology indexes and a human capital variable that grows at a positive constant rate. Therefore, the original technology variables, $A_{t}$ and $B_{t}$, evolve over time at a rate that on average is $\gamma$, but under a cyclical behaviour. In this way, one identifies for the technology variables an evolution process that combines a growth trend and endogenous cycles. 


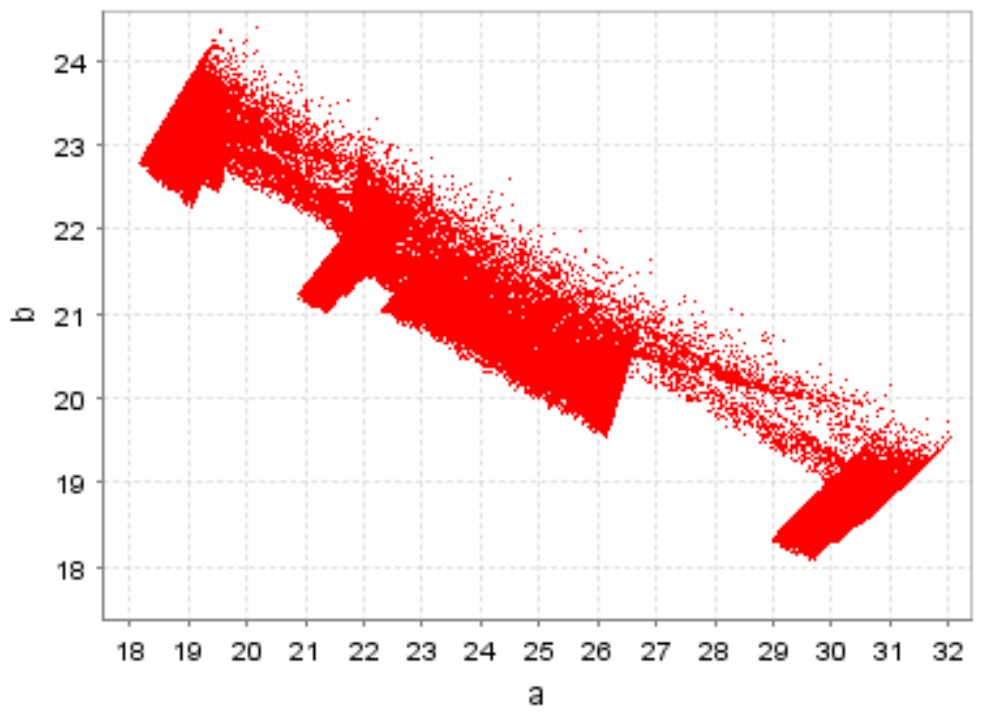

Figure 9 - Attractor $\left(a_{t}, b_{t}\right)$

[parameter values $\left.\psi_{1}=\varphi_{1}=0.5, \psi_{2}=0.95, \varphi_{2}=0.9, \phi=0.7, \mu=0.3, \theta_{1}=1.53, \theta_{2}=2\right]$.

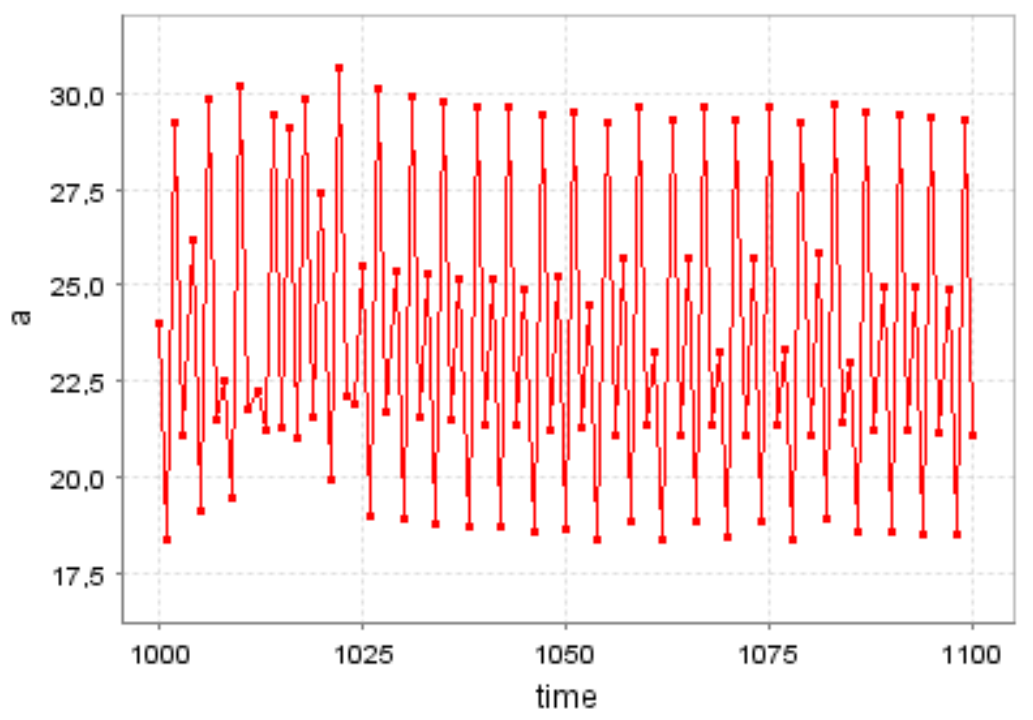

Figure 10 - Time series $a_{t}$.

[parameter values $\left.\psi_{1}=\varphi_{1}=0.5, \psi_{2}=0.95, \varphi_{2}=0.9, \phi=0.7, \mu=0.3, \theta_{1}=1.53, \theta_{2}=2\right]$.

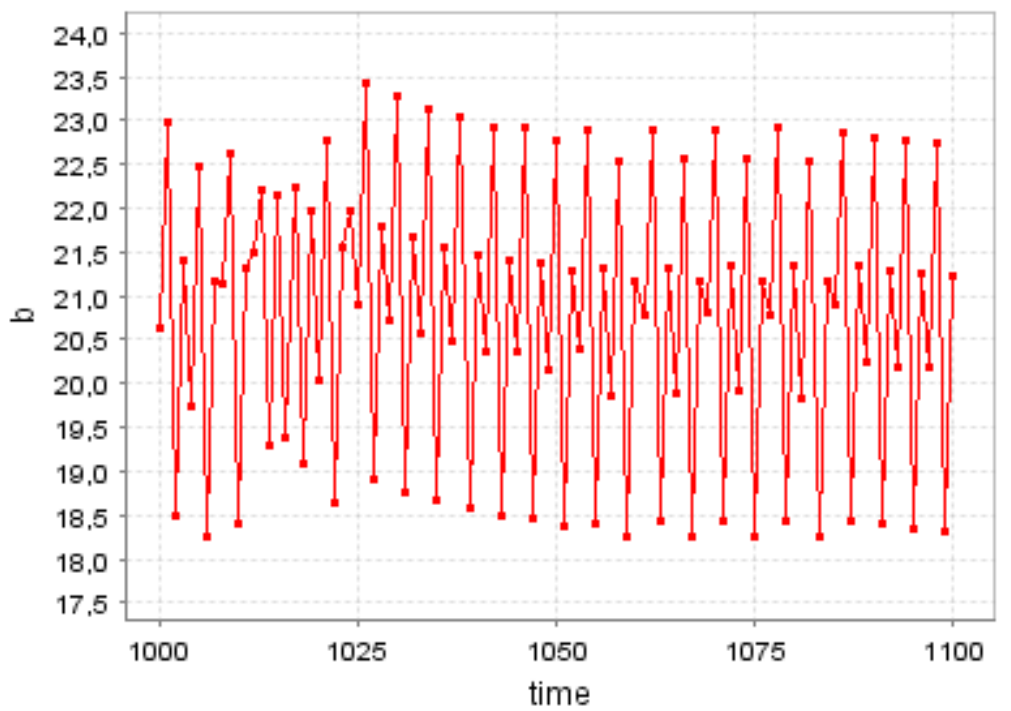

Figure 11 - Time series $b_{t}$. 
[parameter values $\left.\psi_{1}=\varphi_{1}=0.5, \psi_{2}=0.95, \varphi_{2}=0.9, \phi=0.7, \mu=0.3, \theta_{1}=1.53, \theta_{2}=2\right]$.

\subsection{Chaos}

Mathematicians do not agree on an exact definition of chaos. As Sengupta (2003) remarks, nonlinearities in nature and society are pervasive but simultaneously not rigorously understood. Because chaotic motion can arise in multiple and sometimes surprising forms, there is not a universal definition one can apply to chaos, making this phenomenon more a philosophical entity than a mathematical objective notion. For the matter at hand, one will take the most consensual definitions in the context of dynamic systems. These definitions are gathered from the analysis in Medio and Lines (2001), Goenka and Poulsen (2002), Mitra, Nishimura and Sorger (2005) and Gomes (2006b).

We begin by defining a generic discrete dynamic system $x_{t+1}=f\left(x_{t}\right) \in X$, with $X$ a non-empty set and $f$ a map, $f: X \rightarrow X$. For this system, the following two definitions of chaos apply,

Definition 1 - Topological chaos - the dynamic system $x_{t+1}=f\left(x_{t}\right)$ exhibits topological chaos if for this system there is an uncountable scrambled set $(S \subseteq X)$ and a periodic point of a period that is not a power of 2 .

Definition 2 - Ergodic chaos - the dynamic system $x_{t+1}=f\left(x_{t}\right)$ exhibits ergodic chaos if one can identify an absolutely continuous Lebesgue probability measure $v$ on $X$ which is invariant and ergodic under $f$.

A thorough characterization of the properties of chaotic systems is outside the scope of this paper. We just discuss briefly the notions needed to understand the above definitions. After that, we make reference to the most common tool to inquire about the presence of chaos, namely Lyapunov characteristic exponents and present these for various combinations of parameter values in our R\&D model.

The notion of topological chaos is associated to the theorem of $\mathrm{Li}$ and Yorke (1975), which states that any continuous system with a periodic point of period 3 will exhibit chaos. This allows for a direct statement about the presence of chaos in the previously discussed model. As one realizes looking at figures 7 and 8 , there are certain regions in the space of parameters where we clearly identify the presence of cycles of order 3 (and other odd orders). Under the cited theorem, then there must exist also areas 
in the parameter space, for the same system, where complete a-periodicity prevails. Thus, part of the area in white in figures 7 and 8 is not only an area with periodic points of order above 35, but a region of periodicity of no identifiable order, which we associate with the notion of chaos (that is, a simple non rigorous definition of chaos would be linked to the idea that for some combinations of parameter values if we let the deterministic system run for a large number of observations, we will not be able to find any regular pattern of evolution). Definition 1 applies only for an uncountable scrambled set $S$. A rigorous definition of a set with these properties can be found in Mitra, Nishimura and Sorger (2005) and Gomes (2006b). Loosely speaking, set $S$ corresponds to a strange attractor, that is, to an attracting set to which the system may converge in the long run that is not either a fixed point or a periodic point of any identifiable order.

The second definition of chaos is associated with measure theory, that is, to the investigation of the statistical properties of groups of orbits. The ergodic approach to chaos focus on the probabilistic properties of deterministic systems. We will not explore this approach [see Medio and Lines (2001), chapter 9]. To understand definition 2 one should clarify that the probability measure $v$ is invariant if $f$ is a measure preserving map with respect to $v$ (i.e., if, relatively to this measure, sets of a certain size are mapped by $f$ into sets of the same size). The invariant measure $v$ is ergodic if, for every measurable set $V \subseteq X$ satisfying $\{x \in X \mid f(x) \in V\}=V$, it holds that $v(V) \in\{0,1\}$.

The previous definitions characterize chaos in a formal way, however they are difficult to implement in order to identify the true nature of a deterministic dynamic system. Associated to the definition of topological chaos is one of the most widely accepted properties of chaotic systems: sensitive dependence on initial conditions (SDIC). A system displays SDIC if orbits starting from points that are very close but that do not exactly coincide tend to follow different trajectories after a few iterations. Of course, in a deterministic system if the initial state is the same for two trajectories, these trajectories will be identical throughout the time span that is considered, but if initial states are not exactly the same we will have chaotic motion when two trajectories rapidly evolve towards states that are not similar at all.

Because the most common property of chaotic systems is that they tend to display SDIC, the most used instrument to evaluate the presence of chaos in a deterministic system is a measure of exponential divergence of nearby orbits, that is, Lyapunov characteristic exponents (LCEs). The LCEs are analytically defined, for some system of 
order $m$, as the following limit, LCEs $=\lim _{n \rightarrow \infty} \frac{1}{n} \cdot \ln \left\|\prod_{i=0}^{n-1} D f\left(x_{i}\right)\right\|$, where $D f\left(x_{i}\right)$ is a $m \times m$ matrix with elements $\partial f_{j} / \partial x_{l}$, where $f_{j}$ is some function $j$ of the system and $x_{l}$ is some variable $l$ of the system.

In the specific case under analysis, i.e., the R\&D system, there are only two equations, and therefore one may remark that:

i) two LCEs are determinable;

ii) if both LCEs are negative, there is no divergence of nearby orbits. In this case, a fixed point may be observed (obviously, if there is a fixed point nearby orbits do not diverge; on the contrary, they will converge to the same equilibrium value). Periodic points of various orders will correspond as well to a case where LCEs are negative (orbits can converge to one of the $p$ possible long term outcomes, with $p$ the number of periods of the cycle);

iii) a positive LCE signals that nearby orbits exponentially diverge and, thus, the presence of at least one positive LCE relates to the lack of predictability in the system, which is often a good argument to support the presence of chaos.

In table 1, we present Lyapunov exponents for several possibilities regarding parameter values. ${ }^{2}$ All the selected values of parameters reflect situations of chaos, given that one of the LCEs is positive in every case. The reader might want to compare the results in table 1 with figures 7 and 8 to better perceive where regions of chaos arise. Note that the first case in the table is the benchmark case that has allowed drawing the time series in figures 10 and 11. The other eight possibilities just change the value of a given parameter letting the other remain on their benchmark values.

\begin{tabular}{c|cccc|cc} 
& $\theta_{1}$ & $\theta_{2}$ & $\phi$ & $\mu$ & LCE1 & LCE2 \\
\hline$i$ & 1.53 & 2 & 0.7 & 0.3 & 0.245 & -0.056 \\
$i i$ & 0.8 & 2 & 0.7 & 0.3 & 0.311 & -0.050 \\
$i i i$ & 3 & 2 & 0.7 & 0.3 & 0.125 & -0.032 \\
$i v$ & 1.53 & 2.5 & 0.7 & 0.3 & 0.311 & -0.065 \\
$v$ & 1.53 & 5 & 0.7 & 0.3 & 0.387 & -0.038 \\
$v i$ & 1.53 & 2 & 0.75 & 0.3 & 0.180 & -0.052 \\
$v i i$ & 1.53 & 2 & 0.8 & 0.3 & 0.165 & -0.050 \\
viii & 1.53 & 2 & 0.7 & 0.4 & 0.418 & -0.044
\end{tabular}

\footnotetext{
2 The values in the table were also computed using iDMC.
} 

$i x$
1.53
2
0.7
0.8
0.154
$-0.011$

Table 1 - Lyapunov characteristic exponents for several combinations of parameter values $\left[\psi_{1}=\varphi_{1}=0.5, \psi_{2}=0.95, \varphi_{2}=0.9\right]$.

With the computation of LCEs we confirm the results in previous sub-sections, for which the presence of chaos seemed evident. The next step, in the following section, consists in associating the $\mathrm{R} \& \mathrm{D}$ setup that was developed to a growth model, as a way to generate endogenous real business cycles inside a deterministic capital accumulation framework.

\section{Capital Accumulation}

In this section, we introduce a final goods sector. We are interested in addressing the growth implications of bounded technological progress and, therefore, a capital accumulation constraint is added to the analysis. We consider an exogenous saving rate instead of the Ramsey utility maximization framework. The reason is simple. To continue to obtain cycles of various orders as we introduce our technological variables into the final goods sector, it is necessary a growth setup that exhibits stability.

As it is well known, the Ramsey model gives place to a system of two equations, describing the movement of physical capital and consumption through time, that is characterized by saddle-path stability (the system may in fact be reduced to a onedimensional equation describing the time path of the consumption - capital ratio; under standard assumptions, this equation is unstable: for any initial state that does not exactly coincide with the steady state, the defined ratio will diverge from the long term stable locus). The only way to use the consumer optimization problem to characterize growth dynamics with meaningful results, under the technology external effects scenario, would be to consider that the saddle-path is followed in every circumstance. This does not differ significantly from the assumption of an exogenous saving rate.

Take a standard capital accumulation constraint, $K_{t+1}-K_{t}=s K_{t}^{\alpha} \cdot\left(A_{t}^{\beta} B_{t}^{1-\beta}\right)^{1-\alpha}-\delta K_{t}, K_{0}$ given. In this equation, $K_{t}$ defines the available amount of capital in period $t, 0<s<1$ represents the savings rate, $\delta>0$ is the rate of capital depreciation and $\alpha, \beta \in(0,1)$ are elasticity parameters. The production function in this equation is characterized by labour augmenting technological progress (as a simplification, the amount of labour is considered constant and equal to 1). 
Defining $k_{t} \equiv \frac{K_{t}}{H_{t}}$, the capital accumulation constraint can be written in intensive form: $k_{t+1}=\frac{1}{1+\gamma} \cdot\left[s k_{t}^{\alpha} \cdot\left(a_{t}^{\beta} b_{t}^{1-\beta}\right)^{1-\alpha}+(1-\delta) \cdot k_{t}\right]$. Considering reasonable values for the saving rate, for the depreciation rate and for the elasticity parameters, one verifies that the strange dynamics that characterize the technology system are passed to the growth model. To confirm this evidence take a look at the bifurcation diagram in figure 12 , the attractor in figure 13 and the time path of the capital variable in figure 14. This last figure clearly indicates that (for the specific set of parameter values for which the figure is drawn) cyclical motion is present in the process of capital accumulation.

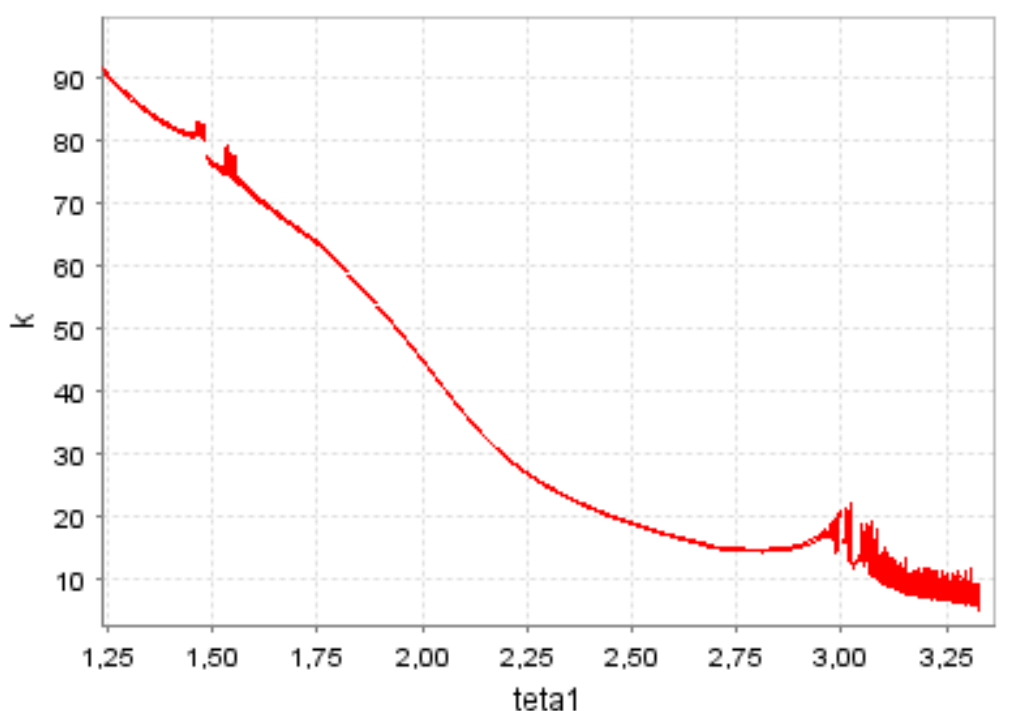

Figure 12 -Bifurcation diagram $\left(\theta_{1}, k_{t}\right)$ [parameter values $\psi_{1}=\varphi_{1}=0.5, \psi_{2}=0.95, \varphi_{2}=0.9, \phi=0.7, \mu=0.3$, $\left.\theta_{2}=2, s=0.25, \alpha=0.25, \beta=0.6, \delta=0.05, \gamma=0.05\right]$.

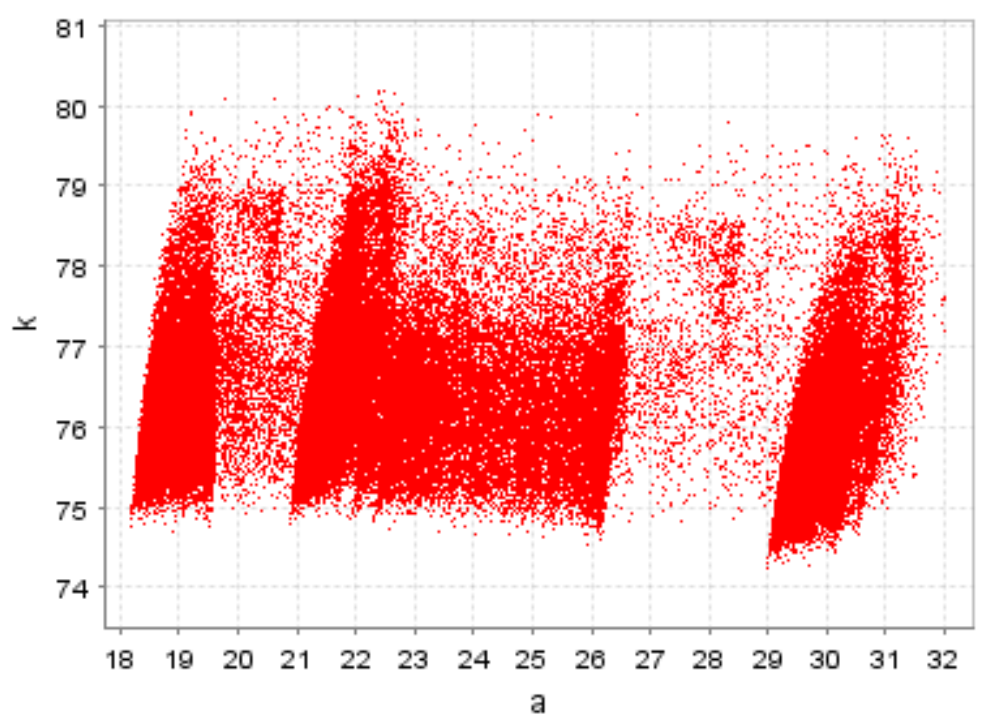

Figure 13 - Attractor $\left(a_{t}, k_{t}\right)$ [parameter values $\psi_{1}=\varphi_{1}=0.5, \psi_{2}=0.95, \varphi_{2}=0.9, \phi=0.7, \mu=0.3, \theta_{1}=1.53$, $\left.\theta_{2}=2, s=0.25, \alpha=0.25, \beta=0.6, \delta=0.05, \gamma=0.05\right]$. 


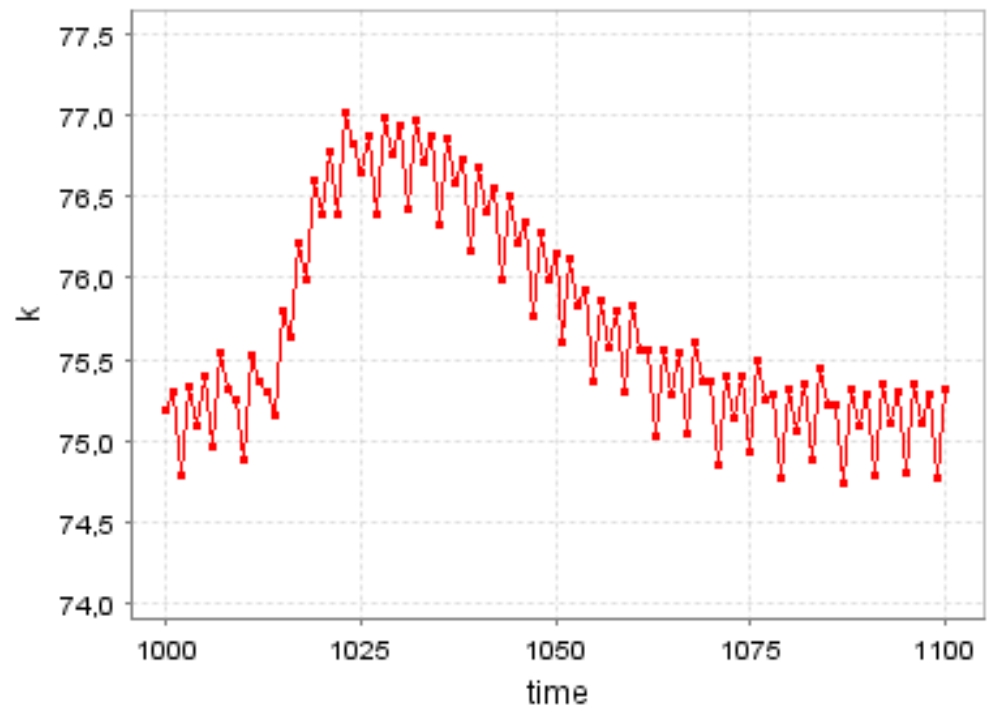

Figure 14 - Time series $k_{t}$ [parameter values $\psi_{1}=\varphi_{1}=0.5, \psi_{2}=0.95, \varphi_{2}=0.9, \phi=0.7, \mu=0.3, \theta_{1}=1.53$, $\left.\theta_{2}=2, s=0.25, \alpha=0.25, \beta=0.6, \delta=0.05, \gamma=0.05\right]$.

Once more, one should emphasize that this is an endogenous growth model, where human capital grows at a constant rate and physical capital, through the influence of the chaotic motion of technology variables, will grow at a rate that is in average constant, although the respective path exhibits fluctuations. Note too that, according to the graphic in figure 14, the model allows to replicate short and long run cycles: within 3-4 periods there are small cycles that co-exist with an overall tendency for larger cycles, that can be depicted for several dozens of observations.

\section{Final Remarks}

The analysis in the preceding sections was motivated by the intuitive idea that strong technological imbalances are useless for economic activity. Because the various technologies are often complementary, consumers and firms in various sectors prefer that technologies be able to keep up with each other. If this does not happen, the economic system tends to produce external effects that allow for a convergence process between R\&D sectors.

The mechanism just described has served to change the traditional two-sector endogenous growth model of innovation and capital accumulation into a framework able to characterize endogenous business cycles. The local analysis allowed for concluding that in the parameters' space we encounter regions of full stability and saddle-path stability, which are separated by a flip bifurcation line. The global dynamic analysis reveals a much more profound set of possible outcomes. Cycles of various orders and a-periodic / chaotic motion describe the interaction between the two R\&D 
sectors that is imposed by the external effects, for different parameter values. Combinations of parameter values leading to chaos are particularly meaningful, indicating that the process through which the outputs of $R \& D$ sectors stay close conducts to an everlasting process of rise and decline relatively to some benchmark average value (that in the case represents a ratio between a technology variable and the level of human capital, this one growing at a constant rate in time).

On a second stage, we have taken the technology indexes time series into a conventional production function for goods, with labour augmenting technical progress. Immediately one verifies that the dynamic properties underlying innovation are passed to a Solow-type capital accumulation constraint and, as a result, endogenous business cycles gain a candidate explanation.

We must emphasize that the obtained results are true under the specific externality functions that were presented. While these serve the purpose at hand, it is true that they are not subject to direct generalization when other types of external effect functions are considered. The undertaken model structure should be interpreted as a possible way to combine the perfectly competitive growth setup (that is very useful to characterize growth trends but that seems unable to jointly describe the fluctuations that occur simultaneously with the growth process), and a kind of 'market imperfection' able to introduce fluctuations. The presence of negative externalities affecting the leading technology sector, as well as positive externalities that make it possible for slow growth innovation sectors to catch up was characterized as a possible mean to combine the long run growth analysis with the short run presence of fluctuations that are determined only by real factors and not by any type of nominal / monetary phenomenon.

\section{References}

Baumol, W. J. and J. Benhabib (1989). "Chaos: Significance, Mechanism, and Economic Applications.” Journal of Economic Perspectives, vol. 3, pp. 77-107.

Benhabib, J. and R. H. Day (1981). "Rational Choice and Erratic Behaviour." Review of Economic Studies, vol. 48, pp. 459-471.

Boldrin, M.; K. Nishimura; T. Shigoka and M. Yano (2001). "Chaotic Equilibrium Dynamics in Endogenous Growth Models." Journal of Economic Theory, vol. 96, pp. 97-132. 
Boldrin, M. and M. Woodford (1990). "Equilibrium Models Displaying Endogenous Fluctuations and Chaos: a Survey." Journal of Monetary Economics, vol. 25, pp. $189-223$.

Bresnahan, T. F.; E. Brynjolfsson and L. M. Hitt (2002). "Information Technology, Workplace Organization and the Demand for Skilled Labor: Firm-Level Evidence.” Quarterly Journal of Economics, vol. 117, pp. 339-376.

Brynjolfsson, E. (1993). "Information Technology and the Productivity Paradox: Review and Assessment." Communications of the ACM, vol. 35, pp. 66-77.

Bullard, J. B. and A. Butler (1993). "Nonlinearity and Chaos in Economic Models: Implications for Policy Decisions.” Economic Journal, vol. 103, pp. 849-867.

Cellarier, L. (2006). "Constant Gain Learning and Business Cycles." Journal of Macroeconomics, vol. 28, pp. 51-85.

Chiarella, C. (1992). "Developments in Nonlinear Economic Dynamics: Past, Present and Future." University of Technology Sydney, School of Finance and Economics working paper $n^{\circ} 14$.

Christiano, L. and S. Harrison (1999). "Chaos, Sunspots and Automatic Stabilizers." Journal of Monetary Economics, vol. 44, pp. 3-31.

Day, R. H. (1982). "Irregular Growth Cycles." American Economic Review, vol. 72, pp.406-414.

Goenka, A. and O. Poulsen (2004). "Factor Intensity Reversal and Ergodic Chaos." Working paper 04-13, Aarhus School of Business, Department of Economics.

Gomes, O. (2006a). "Local Bifurcations and Global Dynamics in a Solow-type Endogenous Business Cycles Model.”, Annals of Economics and Finance, vol. 7, pp. 91-127.

Gomes, O. (2006b). "Routes to Chaos in Macroeconomic Theory." Journal of Economic Studies, forthcoming.

Grandmont, J. M. (1985). “On Endogenous Competitive Business Cycles." Econometrica, vol. 53, pp. 995-1045.

Gunnarsson, G.; E. Mellander e E. Savvidou (2004). "Human Capital is the Key to the IT Productivity Paradox.” Institute for Labour Market Policy Evaluation working paper 2004:13.

Guo, J. T. and K. J. Lansing (2002). "Fiscal Policy, Increasing Returns and Endogenous Fluctuations." Macroeconomic Dynamics, vol. 6, pp. 633-664.

Jones, C. I. (1995). "R\&D-Based Models of Economic Growth." Journal of Political Economy, vol. 103, pp. 759-784. 
Jones, C. I. and J. Williams (2000). "Too Much of a Good Thing? The Economics of Investment in R\&D." Journal of Economic Growth, vol. 5, $\mathrm{n}^{\circ}$ 1, pp. 65-85.

Li, T. and J. Yorke (1975). "Period Three Implies Chaos.” American Mathematical Monthly, vol. 82, pp. 985-992.

Medio, A. and M. Lines (2001). Nonlinear Dynamics: a Primer. Cambridge, UK: Cambridge University Press.

Mitra, T.; K. Nishimura and G. Sorger (2005). "Optimal Cycles and Chaos.” Cornell University, Kyoto University and University of Vienna working paper.

Nishimura, K. and M. Yano (1995). "Nonlinear Dynamics and Chaos in Optimal Growth: an Example.” Econometrica, vol. 63, pp. 981-1001.

Romer, P. M. (1990). “Endogenous Technological Change.” Journal of Political Economy, vol. 98, pp. S71-S102.

Schmitt-Grohé, S. (2000). "Endogenous Business Cycles and the Dynamics of Output, Hours, and Consumption." American Economic Review, vol. 90, pp. 1136-1159.

Sengupta, A. (2003). "Toward a Theory of Chaos." International Journal of Bifurcation and Chaos, vol. 13, pp. 3147-3233.

Solow, R. M. (1987). “We'd Better Watch Out.” New York Times Book Review, July 12, p. 36.

Stutzer, M. J. (1980). “Chaotic Dynamics and Bifurcations in a Macro-Model.” Journal of Economic Dynamics and Control, vol. 2, pp. 353-376. 